\title{
Line Break
}

National Cancer Institute

\section{Source}

National Cancer Institute. Line Break. NCI Thesaurus. Code C80502.

A code that signifies the end of a line. 